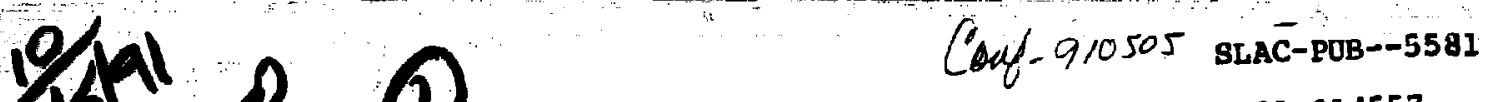 \\ DE91 014557
}

\section{Effect of Wakefields on First Order Transport in the SLC Linac*}

\author{
Chris Adolphsen, Karl L. F. Bane and John T. Seeman \\ Stanford Linear Accelerator Center, Stanford University, Stanford California 94309
}

\section{Abstract}

- The limitation in increasing the bearn current in the SLC linac comes from the emitunce growth caused by wakefields. Simulations of the beam transport that model the wakefield dymamics are being done to study methods to control this growth. To verify the theoretical estimates of the wakefield strengths assumed in these simulations, data were taken which are sensitive to their effect on the first order linac transport. Specifically, the dependence of single beam loading and betatron motion on beam current was measured in the range of $0.5 \cdot 10^{10}$ to $3.5 \cdot 10^{10}$ electrons per bunch. This paper presents these data together with comparisons to results from simulations.

\section{INTRODUCTION}

The SLC - Jinac accelenates bunchies of particles from an energy of about $1 \mathrm{GeV}$ to $47 \mathrm{GeV}$ along a $3 \mathrm{~km}$ FODO latice which contains 275 quadrupole magnets. The elements in the linac are illustrated in Figure 1. Along the linac, the phase advance of the FODO cells vary $\left(90^{\circ} \rightarrow 40^{\circ}\right)$ as does the quadrupoic spacing $(3 \mathrm{~m} \rightarrow 12 \mathrm{~m})$. The beam is accelerated in disk-loaded waveguide structures which have an average iris radius (a) of $1.1 \mathrm{~cm}$ and a cell length of $3.5 \mathrm{~cm}$. The longitudinal $\left(W_{L}\right)$ and transverse $\left(W_{T}\right)$ wakefields generated in these strucures have been computed for the $m=0$ and $m=1$ modes, respectively [1]. They are plouted in Figure 2 as a function of the longitudinal separation ( $\Delta s$ ) of the paricle generating the field, and a test particle. Note that neither function depends on the transverse position of the test particle.

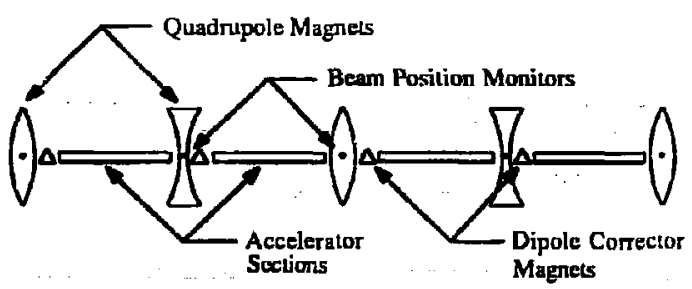

Figure 1. Segment of the SLC linac.

For an ensemble of particles, the wakeficld interactions couple the particle motions. The effect can be described by treating the beam as serics of longitudinal slices denoted by their longitudinal positions (s) relative to the bunch center. The energy loss and transverse angular kick per unit length $(z)$ of each stice due to the waketiclds are

$$
\frac{d E}{d z}(s)=e I \int_{s}^{\infty} \rho\left(s^{\prime}\right) W_{L}\left(s^{\prime}-s\right) d s^{\prime}
$$

- Work supported by Deparment of Energy contrac DE-AC03-76SF00515.

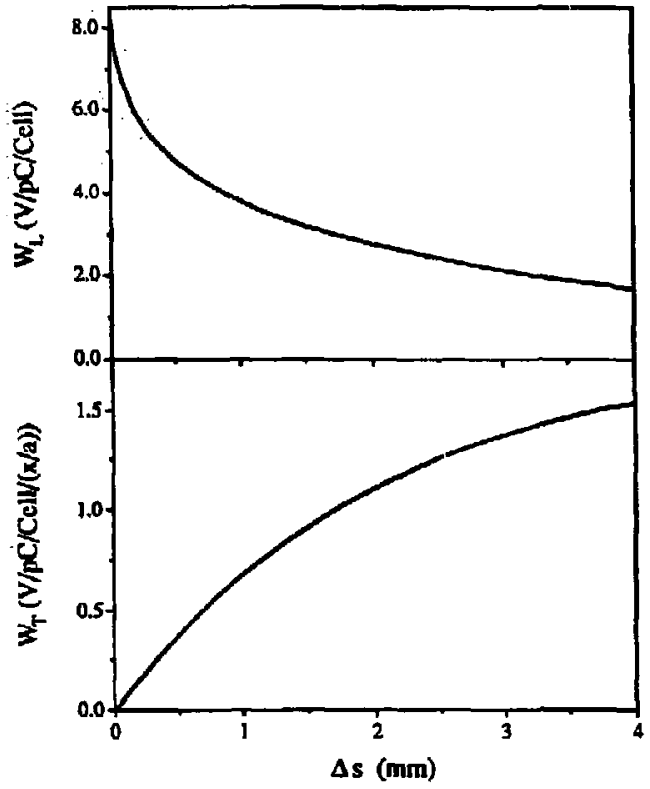

Figure 2. Longitudinal $\left(W_{L}\right)$ and transverse $(W T)$ wakefield functions. $W_{T}$ is linear in the transverse displacement (x/a) of the leading particle.

and

$$
\frac{d \theta}{d z}(s)=e^{2} \frac{I}{E(s)} \int_{s}^{\infty} p\left(s^{\prime}\right) W_{T}\left(s^{\prime}-s\right) x\left(s^{\prime}\right) d s^{\prime}
$$

where $E(s)$ is the slice energy, $I$ is the beam current, $x$ is the uransverse slice position relative to the waveguide axis, and $p(s)$ is the longitudinal charge density (unit normalization). The average energy loss and kick angle are computed by integrating these expressions over the bunch length profile.

Equation 1 shows that the beam loading is independent of the trajectories of the beam slices. Thus, a measurement of just the beam energy as a function of beam current is needed to gauge the longitudinal wakefield strength. The situation is more complicated for the angular kicks because the kick angle of one slice depends on the positions of all slices upstream of it. For the beam currents considered here, however, this is a weak effect in that the differential motion of the slices caused by the wakcficld kicks over a distance of an oscillation wavelength is small compared to the oscillation amplitude. As described below, this condition can be exploited to infer the strength of the transverse wakefields from their perturbation on betatron motion.

EICTRIJUTICNY CF THIS DOCURMEHT IS UNLIMITED 


\section{BEAM PROPERTES}

To do either of these analyses requires a knowledge of the longitudinal bunch profile. In the production of the linac bunches, this shap: is determined by the profile of the bunch extracted from the damping ring, and by the compression that occurs in the ring-10-linac transport line [2]. For the data taken for this study, the bunches are expected to be nearly Gaussian in shape and have an rms length $\left(\sigma_{\boldsymbol{t}}\right)$ which depends on current as

$$
\sigma_{1}=.61+.12 \cdot 1+.0076 \cdot 1^{2}
$$

where $\sigma_{8}$ is in units of $\mathrm{mm}$ and $\mathrm{l}$ is in units of $10^{10}$ electrons.

Another beam property that affects betatron motion is the energy spread of the beam. The component of energy correlated with $s$ results from the beam loading and the sinusoidial shape of the RF accelerating waveform. The latuer contribution can be computed by summing the energy gains $\Delta E_{i}$ from each klystron $i$. These have the form

$$
\Delta \mathrm{E}_{\mathrm{i}}=\Delta \mathrm{E}_{\mathrm{O}_{\mathrm{i}}} \cdot \cos \left(\phi_{\mathrm{kxs} i}+\phi_{\mathrm{i}}+2 \pi s / \lambda_{\mathrm{RF}}\right)
$$

where $\Delta E_{O_{i}}$ is the 'no-load' energy gain of the klystron, $\lambda_{R F}$ is the RF wavelength $(105 \mathrm{~mm}), \phi_{0}$ is a phase adjustment common to all klystrons, and $\phi_{\mathrm{ans}}$ is a phase used to generate an energy spread to help cancel the variation in the transverse wakefield kicks along the bunch [3]. The BNS phases are constrained by the maximum energy gain possible in the linac, and by the requirement of a small energy spread at the end of the linac. As a tradeoff, a value of $-20^{\circ}\left(15^{\circ}\right)$ is used in the first third (tast two thirds) of the linac. Operationally, a global phase adjustment $\left(\phi_{0}\right)$ is made to minimize the energy width when the beam current is changed. As an example of these contributions, Figure 3 shows the computed rms energy width profile along the linac for $I=3.0 \cdot 10^{10}$. A global phase of $2^{\circ}$ was assumed which corresponds to the value expected for the data taken for this study. The energy spread within any slice of the beam is roughly $1 \% / E$ where $E$ is the beam energy in $\mathrm{GeV}$. This component can be ignored for this analysis without significantly changing the results.

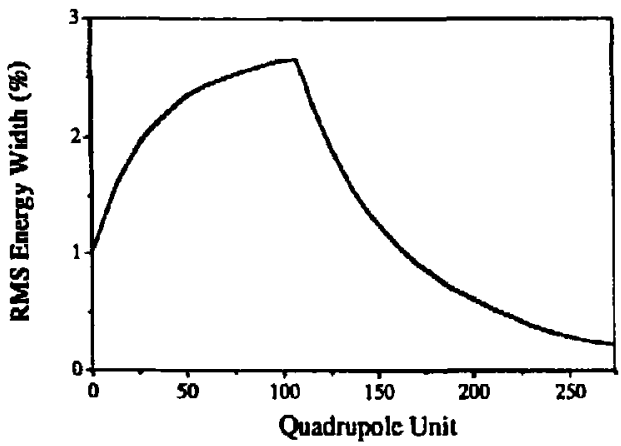

Figure 3. Predicted rms energy width profle along the linac for a current of $3.0 \cdot 10^{10}$ electrons per bunch.

\section{SINGLE BEAM LOADING}

To measure the strength of the longitudinal wakefields, the beam energy at the end of the linac was recorded for sevcn beam currents in the range of $0.5 \cdot 10^{10}$ to $3.5 \cdot 10^{10}$ electrons per bunch. No other tuning of the linac was donz during data taking. Because only relative energy changes are well measured, a systematic adjustment was made to the values to compule the energy loss relative to the zero current energy. This adjustment was determined by finding the best overall match of the measured energy losses to the values from a simulation of these measurements. The results, expressed as fractional energy losses ( $\triangle E / E$ ) at the end of the linac, are shown in Figure 4 for both the data and simulation.

The good agreement between the measured and simulated energy losses indicates that the longitudinal wakefields are well modeled. In the simulation, the bunch length profile was assumed to be Gaussian with a sigma given by Equation 3 . The shaded area in the plot shows the range of predictions if the bunch length is changed within $+1-20 \%$ of the nominal values. The small nonlinearily in the energy loss arises mainly from the assumed bunch length dependence on current. A few pereent nonlinearily also results from the phase shiff caused by the beam loading in the acceleration section used for bunch compression in the ring-to-linac retum line [4].

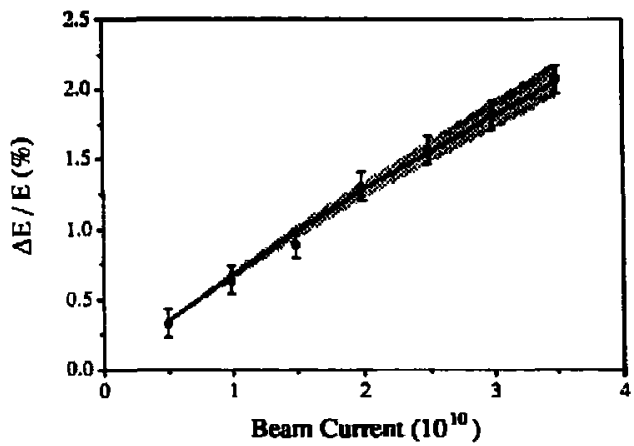

Figure 4. Measured (circles) and simulated (solid line) fractional energy loss as a function of beam current. The shaded region is described in the text.

\section{BETATRON OSCILLATIONS}

The measurement of the uransverse wakeficld strength uses the approximation that the variation of the slice positions within a bunch undergoing betatron motion can be ignored relative to the mean oscillation amplitude. This approximation, which is independent of the amplitude of the oscillation, is valid in only cerain regimes which must be determined through a simulation of the beam transport. In this regime, the equations of motion can be simplified by removing the $x\left(s^{\prime}\right)$ term from the integral in Equation. 2. The average kick angle in this approximation, denoted here by a subscripl $A$, is

$$
\left\langle\frac{\mathrm{d} \theta}{\mathrm{d} z}\right\rangle_{\mathrm{A}} \equiv \alpha \frac{\mathrm{I}}{\mathrm{E}}(\mathrm{x})
$$

where

$$
\alpha=e^{2} \int_{-\infty}^{\infty} \int_{s}^{\infty} \rho(s) \rho\left(s^{\prime}\right) W_{T}\left(s^{\prime}-s\right) d s d s^{\prime}
$$

and $E$ is the mean beam energy. This relation has a quadrupole-like form in that the kick angle depends lincarly 
on beam position. However. the kick is always away from the waveguide axis which leads to a lengthening of the betatron oscillation waveleagth. This effect makes it appear that the beam energy is larger, which is opposite to that of beam loading.

To check the validity of this approximation, and to model any linac measurements involving betatron oscillations, a simulation program was written which treats the beam as a series of slices and transpors them through the linac elements in a step wise manner. The bearn profile, which was assumed Gaussian, was represented by 41 slices covering $+1-3$ timis the ms bunch length as given by Equation 3 . The effect of wakefields was included by integrating Equations 1 and 2 for each slice over the lengths of the acceleration sections.

With this program, betatron oscillations beginning at the upstream end of the linac were simulated to check the approximation used to derive Equation 5. Specifically, the ratio

$$
R \equiv\left\langle\frac{d \theta}{d z}\right) /\left(\frac{d \theta}{d z}\right\rangle_{A}
$$

was computed at cach beam position monitor (BPM) location using Equations 2 and 5 with the substitution

$$
x(s) \rightarrow \bar{x}(s) \cos \left(\phi(s)-\phi_{M}\right)+\bar{X}^{\prime}(s) \sin \left(\phi(s)-\phi_{M}\right)
$$

to make $R$ insensitive to the phase of the oscillation. Here $\bar{x}(s)$ and $\bar{x}^{\prime}(s)$ are the normalized position and slope coordinates of the slice, and $\phi(s)-\phi_{M}$ is the phase of the slice relative to the mean phase of the beam. If the motion of the slices remains coherent $\mathbf{R}$ is unity, while for complete decoherence, $R \rightarrow \infty$. From the simulations, it was found that $R$ remains within $+/-25 \%$ of unity in the first third of the linac for the beam currents being considered. Given this somewhal arbitrary measure of the goodness of the kick angle approximation, it was decided to limit the data analysis to only this region of the linac. Within this region, $R$ is larger on average at low currents than at high currents although the mechanism for this is not clear.

The effect of the average kick angle described by Equation 5 can be incorporated into a first order transport matrix (in $x$ and $x^{\prime}$ ) representation of the linac by including a matrix for the acceleration sections which is an integration of the kick angles over the lengths (L) of the seclions. For the case of no acceleration, the $2 \times 2$ drift transpon matrix is modified as

$$
\left[\begin{array}{ll}
1 & \mathrm{~L} \\
0 & 1
\end{array}\right] \rightarrow\left[\begin{array}{ll}
1 & \mathrm{~L} \\
0 & 1
\end{array}\right]+\alpha \frac{\mathrm{IL}^{2}}{2 \mathrm{E}}\left[\begin{array}{cc}
1 & \mathrm{~L} / 3 \\
2 / \mathrm{L} & 1
\end{array}\right]
$$

to first order in $\alpha$ (nole that this correction does not apply to the propagation of beam ellipses). With this substitution, the 1,2 elements of the iransport matrices computed from a corrector magnet to all downstream BPM positions should approximately match the shape of a betatron oscillation induced using that corrector. Conversely, a fit to betatron oscillation data can be made to extract the beam current dependence as specified in Equation 9. This has the advantage of allowing the effect of approximations to be absorbed into the fit current.

For this purpose, oscillation data were taken at the seven current settings used when measuring the beam loading. One corrector was used to generate an oscillation in the horizontal plane near the beginning of the linac and the resulting BPM difterence orbit was recorded logether with the magnets settings and the computed energy profile along the linac. The largest error in reconstructing the lattice from this information comes from the uncertainty in the energy scale. This factor, however, can be estimated by fitting the data to a single particle trajectory with the energy scale included as a variable [5]. For this analysis, the scale factor was adjusted so the fit to the $0.5 \cdot 10^{10}$ data yielded the same result as the fit to a simulation of these data. The lowest current data were chosen because they are least affected by wakefields. A correction of about $2 \%$ was found and was applied to all seven data sets. A correction for beam loading for each current was then made using the results in Figure 4. Finally, each BPM difference orbit was fit assuming a single particle trajectory, but including the correction in Equation 9. The initial position and slope of the trajectory was allowed to vary in the fit, as was the beam current (In) entering the correction.

The same procedure was applied to simulated BPM oscillation data for the seven beam currents. No errors were included in the BPM readings, which in the data can be ignored relative $w$ the amplitude of the oscillations. As an example of the results, Figure 5 shows the measured and simulated $B P M$ values, and the resuiting fits for $I=3.0 \cdot 10^{10}$. In each plot there is some systematic disagreement between the fit and the BPM readings. However, an exact fit is not expected even without wakefields because a single particle trajectory does not account for incoherence elfects. For the simulation, the rms of the fit residuals are $10 \%$ to $20 \%$ of the rms of the BPM values. In the data, they are $20 \%$ to $30 \%$ for all but the lowest two current settings where they are $37 \%$ and

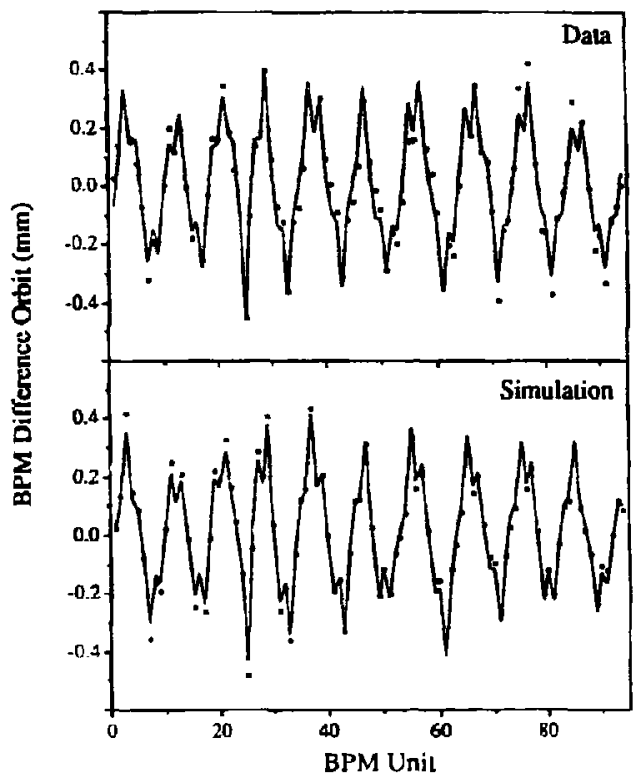

Figure 5. Measured and simulated BPM oscillations (circles) and the resulling fits (solid lines) for $1=3.0 \cdot 10^{10}$. 
$34 \%$, respectively. This may be due to the fact that the lower current data is more sensitive to errors in the energy profile along the linac.

The results from the current fits are shown in Figure 6 for both the data and simulation. Note that the good agreement for $I=0.5 \cdot 10^{10}$ is the result of the energy scale factor correction to the data. The error bars on the data correspond to a $0.2 \%$ uncertainty in the energy scale, which is a rough estimate of the error given the method in which the scale was determined. However, other types of energy profile errors are not included. The shaded region represents the range of predictions for $a+1-3^{\circ}$ change in the global phase $\left(\phi_{0}\right)$ or a +1 $20 \%$ variation in the bunch length. The phase change was made so it affected only the energy spread and not the mean energy gain.

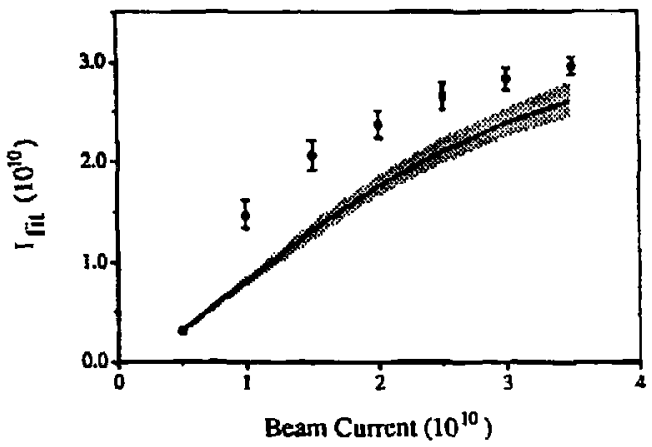

Figure 6. The fit value for the beam current from measured (circles) and simulated (solid line) BPM difference orbits as a function of the actual beam current. The shaded region is described in the text.

Given these systematic errors, the values of Jri for the dnta are still larger than expected. Scaling the strength of the transverse wakefield function improves the agrecment, although a $60 \%$ increase is necessary due in part to the decrease in the results from the simulation that also occurs with stronger wakefields. Other possible causes of the difference are errors in the energy profile along the linac, a non-Gaussian bunch length profile, or a:value of $R$ for the bean at the beginning of the oscillation which is not unity as assumed in the simulation. Repeating the measurements with a more careful setup of the linac may help eliminate some of these possibilities. In any case it is interesting to note the leveling-off of the Init values at large currents. This is most likely due to the fact that $\mathbf{R}$ decreases at larger currents as was noted previously. Equation 5 thus overestimates the average wakefield kick angle as the current increases.

To get a sense of the size of the wakcield effect, an equivalent energy change was computed for each FODO cell of the linac that cancels the change in phase advance per cell due $w$ the transverse wakerields. That is, the change in the 1,1 and 2,2 elements of the cell transport matrix from the substitution in Equation 9 is canceled by a decrease in energy of

$$
\Delta E_{w} \equiv 2 \alpha I\left(1-K^{2} L^{2} / 12\right) / K^{2}
$$

where $K$ is the inverse focusing strength of the quadrupoles in the cell. For $I=3.0 \cdot 10^{10}$, the required fractional encrgy dccrease is plotued in Figure 7 for the region of the linac used in the oscillation analysis. Compensation of the average wakefield kick angle would thus require energy shifts as large as 10\%. It is interesting to note that a similar calculation can be done to compute the energy change as a function of $s$ to make the phase advance independent of $\mathbf{s}$ (this is sometimes referred to as autophasing). In this case, the resulting rms energy spread is similar in magnitude to the mean energy shif shown in Figure 7. Comparing Figures 3 and 7 shows that the energy spread in the upstream end of the linac is approximately that required for this condition. This agreement is consisten! with the fact that $\mathbf{R}$ remains close to unity in this region.

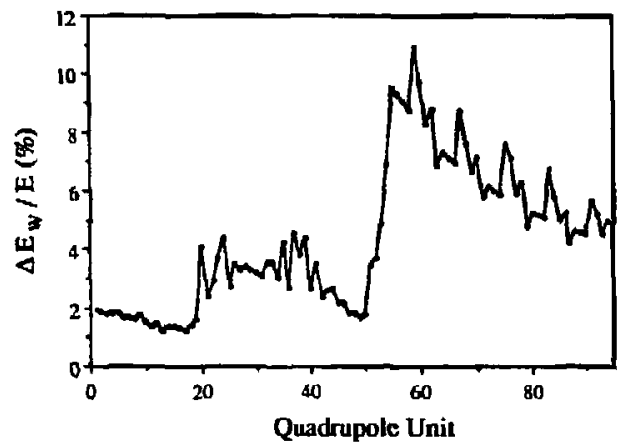

Figure 7. The fractional energy decrease required to cancel the effect of the transverse wakefields on the phase advance per cell as a function of quadrupole unit for $I=3.0 \cdot 10^{10}$. The discontinuities in the values are due to the uneven spacing of the quadrupoles.

\section{SUMMARY}

The measurements of beam loading are well modeled which gives one confidence in the assumed strength of the longitudinal wakefields. Measuring the transverse wakelicld strength is more complex, requiring a perturbation treatment of their effect on betatron motion. The fits for the beam current from measured oscillation data show a qualitative agreement with the results from simulated data. Further measurements of this type with a better control of systematic errors are necded to make more quantitative conclusions.

\section{V1. REFERENCES}

[1] K. Bane and P.B. Wilson, Proc. of the $l J^{\text {th }}$ Int. Conf. on High Energy Accelerators, CERN (1980), 592.

[2] K. Bane, "Simulating Longitudinal Phase Space in the SLC, from the Damping Rings to the Final Focus," SLAC/AP-80 (Stptember 1990).

[3] V. Balakin, A. Novokhatsky and V. Smimov, Proc. of the $12^{\text {th }}$ Int. Conf. on High Energy Acceleralors, Fernilab (1983), 119.

[4] This effect was pointed out to us by F.J. Decker.

[5] T. Himel and K. Thompson, "Energy Measurements from Bctalion Oscillations," SLAC-PUB-4917 (March 1989). 


\section{DISCLAMER}

This report was prepared as an account of work sponeored by an agency of the United States Government. Neither the United States Government nor any agency thereof, nor any of their employes, makes any warranty, express or inplied, or assumes any legal liability or nesponsibility for the eccurncy, completeness, or usefulness of any information, apparatus, product, or procest disclosed, or represents that its use would not infringe privalely owned rights. Reference berein to any spocife commercial product, proces, or service by trade anme trademark, minufacturer, or otherwise does aot necessarily constitute or imply its endorsement, recommendation, of favoring by the Uaited States Governmest or any agency thereof. The views and opinions of authors expresed bercin do not necussarily state or reflect those of the United States Government or any agency thereof. 